\title{
Efeito da cobertura de céu na assimilação de $\mathrm{CO}_{2}$
}

\author{
Effect of sky cover on $\mathrm{CO}_{2}$ assimilation
}

Efecto de la cubierta del cielo sobre la asimilación de $\mathrm{CO} 2$

Recebido: 01/10/2021 | Revisado: 08/10/2021 | Aceito: 13/10/2021 | Publicado: 16/10/2021

Enzo Dal Pai

ORCID: https://orcid.org/0000-0002-0570-8644 Universidade Estadual Paulista, Brasil E-mail: enzo-dal.pai@unesp.br

\begin{abstract}
Resumo
O objetivo deste trabalho foi estudar o fluxo de $\mathrm{CO}_{2}$ no ambiente e sua relação com a cobertura de céu e natureza da radiação solar, na busca de uma relação matemática entre estas variáveis. Objetivou-se também propor um modelo empírico que correlacione radiação solar com fluxos de $\mathrm{CO}_{2}$ (e consequentemente fotossíntese e produtividade vegetal). Dados de fluxos de $\mathrm{CO}_{2}$ foram obtidos de satélites, de duas bases: a base "PML_V2" e a base "MODIS17A2H". A produção diária bruta de carbono apresentou relação com a radiação solar difusa. Os dados de fluxos de $\mathrm{CO}_{2}$ obtidos junto ao programa "PML_V2" se ajustaram melhor aos dados de radiação solar de BotucatuSP. Os dados do programa "MODIS17A2H" carecem de mais estudos, principalmente em sua parametrização, que envolve elementos de vegetação local. A equação multivariada obtida para estimativa da produção primária de carbono GPP em função da radiação solar difusa, o valor Kt o mês apresentou elevados valores de performance, com $r$ $=0.88, \mathrm{MBE}=-6,99 \%$ e RMSE $=16,70 \%$.
\end{abstract}

Palavras-chave: $\mathrm{CO}_{2}$; Radiação solar difusa; Produção primária bruta; PML_V2; MODIS17A2H.

\begin{abstract}
This work aimed to study the $\mathrm{CO} 2$ flux in the environment and its relationship with the sky coverage, in search of a mathematical relationship between these variables. It was also aimed to propose an empirical model that correlates solar radiation with $\mathrm{CO}_{2}$ fluxes (and consequently photosynthesis and plant productivity). $\mathrm{CO}_{2}$ flux data were obtained from satellites, from two bases: the base "PML_V2" and the base "MODIS17A2H". The gross daily production of carbon was related to diffuse solar radiation. The $\mathrm{CO}_{2}$ flux data obtained from the "PML_V2" program better fit the solar radiation data from Botucatu-SP. The data from the "MODIS17A2H" program needs further studies, especially in its parameterization, which involves elements of local vegetation. In the multivariate equation obtained to estimate the gross primary production of GPP as a function of diffuse solar radiation, the Kt value per month presented high performance values, with $\mathrm{r}=0.88, \mathrm{MBE}=-6.99 \%$ and $\mathrm{RMSE}=16.70 \%$.
\end{abstract}

Keywords: $\mathrm{CO}_{2}$; Difuse solar radiation; Gross primary production; PML_V2; MODIS17A2H.

\section{Resumen}

El objetivo de este trabajo fue estudiar el flujo de CO2 en el ambiente y su relación con la cobertura del cielo y la naturaleza de la radiación solar, en busca de una relación matemática entre estas variables. También se pretendía proponer un modelo empírico que correlacione la radiación solar con los flujos de CO2 (y en consecuencia la fotosíntesis y la productividad de las plantas). Los datos de flujo de CO2 se obtuvieron de satélites, a partir de dos bases: la base "PML_V2" y la base "MODIS17A2H". La producción diaria bruta de carbono se relacionó con la radiación solar difusa. Los datos de flujo de CO2 obtenidos del programa "PML_V2" se ajustan mejor a los datos de radiación solar de Botucatu-SP. Los datos del programa "MODIS17A2H” necesitan más estudios, principalmente en su parametrización, que involucra elementos de la vegetación local. En la ecuación multivariante obtenida para estimar la producción primaria de carbono GPP en función de la radiación solar difusa, el valor de Kt por mes presentó valores de alto desempeño, con $r=0.88, \mathrm{MBE}=-6.99 \%$ y $\mathrm{RMSE}=16.70 \%$.

Palabras clave: CO2; Radiación solar difusa; Producción primaria bruta; PML_V2; MODIS17A2H.

\section{Introdução}

Estudos sobre a biomassa tem se multiplicado pelo mundo nos últimos anos. No Brasil essa área de estudo também cresce vigorosamente. A biomassa apresenta grande poder estratégico na geração de energia, cuja demanda mundial não para de crescer (Araújo, Higuchi \& Carvalho Jr, 1996). Enquanto a demanda energética mundial cresce, a oferta energética 
oferecida pelos tradicionais combustíveis fósseis começa a sofrer restrições no seu uso (e expansão) devido ao seu caráter nãorenovável e deletério ao ambiente (e possivelmente nas anomalias/mudanças climáticas).

$\mathrm{O} \mathrm{CO}_{2}$ é peça fundamental no entendimento energético da biomassa. Plantas captam o $\mathrm{CO}_{2}$ do ar, e juntamente com a radiação solar, conseguem sintetizar a glicose, molécula com 6 átomos de carbono, precursora de todos os outros carboidratos (Pallardy, 2008; Salisbury, 2012, Zhang et al., 2019). A radiação solar não é perdida, mas absorvida e armazenada na forma de energia química. Essa captação de $\mathrm{CO}_{2}$ realizada pelas plantas, em nível global, apresenta grande magnitude, representado pela retirada de massa de $\mathrm{CO}_{2}$ atmosférico e assimilação de massa de $\mathrm{CO}_{2}$ dentro de tecidos vegetais (Taiz \& Zeiger, 2016) (a captação de $\mathrm{CO} 2$ atmosférico é chamada de fixação do $\mathrm{CO}_{2}$ e o conhecimento de sua magnitude tem grande importância).

Porém, além de assimilar o $\mathrm{CO}_{2}$, plantas também precisam respirar: oxidar a glicose e liberar 6 átomos de carbono para conseguir sua energia e manter seus processos fisiológicos (Taiz \& Zeiger, 2016). É um fluxo contrário ao da assimilação do $\mathrm{CO}_{2}$. Logo plantas retiram $\mathrm{CO}_{2}$ da atmosfera e também devolvem $\mathrm{CO}_{2}$ para a atmosfera. Com esses dois fluxos é possível se estabelecer uma diferença entre eles: um saldo. Este saldo tende a ser positivo de dia (mais $\mathrm{CO}_{2}$ está sendo absorvido que liberado) e negativo durante a noite (sem assimilação de $\mathrm{CO}_{2}$, ocorre apenas liberação de $\mathrm{CO}_{2}$ ). Também vale lembrar que nesses estudos de fluxo de $\mathrm{CO}_{2}$ deve-se sempre considerar também a respiração dos organismos vivos do solo, que contribuem com a maior liberação de $\mathrm{CO}_{2}$ para a atmosfera (Araújo et al., 1999; Salisbury, 2012).

Visto que a radiação solar exerce influência nesses fluxos e nesse saldo, há necessidade de estudos sobre a disponibilidade energética solar e os fluxos de $\mathrm{CO}_{2}$ no ambiente (Yanni et al., 2014). E quando se discute a disponibilidade energética não se deve pensar apenas no valor absoluto da radiação solar, mas sim na sua natureza e distribuição espacial/sazonal (Zhang, Zhang \& Zhoub, 2000).

Diversos pesquisadores já estão trabalhando com medidas de fluxos de $\mathrm{CO}_{2}$ na atmosfera e radiação solar (Alton, North \& Los, 2007; Brodersen, Vogelmann \& Gorton, 2008; Freedman, Fitzjarrald \& Sakai, 2001; Gu, Fuentes, Shugart and Black, 1999; Jing et al., 2010; Matthew, Letts, Lafleur \& Roulet, 2005; Min \& Wang, 2008; Oliphant, et al., 2011; Roderick, Farquhar, Berry \& Noble, 2001; Yanni et al., 2014). A grande linha de pesquisa a ser seguida é o estudo da radiação solar global difusa (Hd) e da radiação solar espectral fotossinteticamente ativa (RFA ou PAR, acrônimo em inglês de "photossintetically active radiation"). A radiação solar difusa é a fração da radiação solar que por qualquer processo físico já sofreu interferência da atmosfera (Alton et al., 2007; Dal Pai, Escobedo, Correa, Dal Pai \& Santos, 2014; Erbs, Klein \& Duffie, 1982). É a típica fração da radiação solar de dias encobertos, nublados ou chuvosos (nestes dias a radiação sofre espalhamento causado pelas nuvens). A radiação $P A R$ é a fração absorvida pelas plantas para o processo de fotossíntese. É possível se medir a fração PAR difusa, que é a radiação solar que está dentro do espectro visível e que já passou por algum processo atmosférico de espalhamento (Ren, He, Zhang \& Yu, 2018; Yamashita \& Yoshimura, 2019).

A radiação solar difusa apresenta a propriedade de ser multidirecional. Por ter sido espalhada, é dito que após o espalhamento, seus vetores de energia são espalhados em todas as direções (Dal Pai et al., 2014; Jacovides, Assimakopoulos \& Tymvios, 2006; Jacovides, Tymvios, Assimakopoulos \& Kaltsounides, 2007).

Este espalhamento garante que a radiação solar difusa apresente um fluxo energético em todos os sentidos, mesmo de cima para baixo ou de baixo para cima. Multirreflexões na atmosfera e no solo garantem que um feixe de energia solar difusa seja capaz de atingir o tecido foliar fotossintético presente na parte de baixo das folhas, aumentando sua eficiência no uso da energia. Diversos pesquisadores atuam nessa área para tentar entender a relação da radiação solar difusa, a cobertura do céu e o fluxo de $\mathrm{CO}_{2}$ no ambiente (Alton et al., 2007; Araújo et al., 1996; Brodersen et al., 2008; Freedman et al., 2001; Gu et al., 1999; Jing et al., 2010; Matthew et al., 2005; Min \& Wang, 2008; Oliphant et al., 2011; Roderick et al., 2001; Yamashita \& Yoshimura, 2019; Yanni et al., 2014; Zhang et al., 2000, Zhang et al., 2019). 
O objetivo deste trabalho é estudar o fluxo de $\mathrm{CO}_{2}$ no ambiente e sua relação com a cobertura de céu e natureza da radiação solar, na busca de uma relação matemática entre estas variáveis. Objetiva-se também propor um modelo empírico que correlacione radiação solar com fluxos de $\mathrm{CO}_{2}$ (e consequentemente fotossíntese e produtividade vegetal). Com estas informações será possível se melhorar modelos de produtividade potencial de biomassa, importantes no planejamento, gestão e operação de grandes sistemas de co-geração de energia. Será possível se fazer estudos da série histórica climatológica sobre anos atípicos de seca ou de grandes chuvas (menor ou maior nebulosidade, respectivamente) e como interferem no rendimento da produção da biomassa e na operação de sistemas de co-geração.

\section{Metodologia}

O local do experimento é a Estação Meteorológica da UNESP de Botucatu, SP (latitude $22^{\circ} 50^{\prime} \mathrm{S}$; longitude $48^{\circ}$ 25 W; altitude 786m). Pela classificação climática de Köppen o clima desta região é o Cwa, caracterizado por ser o temperado quente (mesotérmico), com chuvas no verão e seca no inverno, com a temperatura média do mês mais quente superior a $22{ }^{\circ} \mathrm{C}$ (Dal Pai et al., 2014). O dia mais longo do ano tem 13,4 horas no mês de dezembro, e o mais curto tem 10,6 horas no mês de junho. A série climática de 30 anos é apresentada na Figura 1. O mês de fevereiro é o mais quente do ano com temperatura média de $23,2{ }^{\circ} \mathrm{C}$, enquanto que o mês de julho é o mais frio do ano com temperatura média de $17,1^{\circ} \mathrm{C}$. Fevereiro é o mês mais úmido do ano com valores de umidade em torno de 78,2\% e agosto é o mês mais seco com percentuais em torno de 61,80\% (Dal Pai et al., 2014).

Figura 1: a, b, c. Series Climáticas da temperatura(a), Umidade(a), Nebulosidade(b), Precipitação(c) do período de 1970 a 2000 em Botucatu.
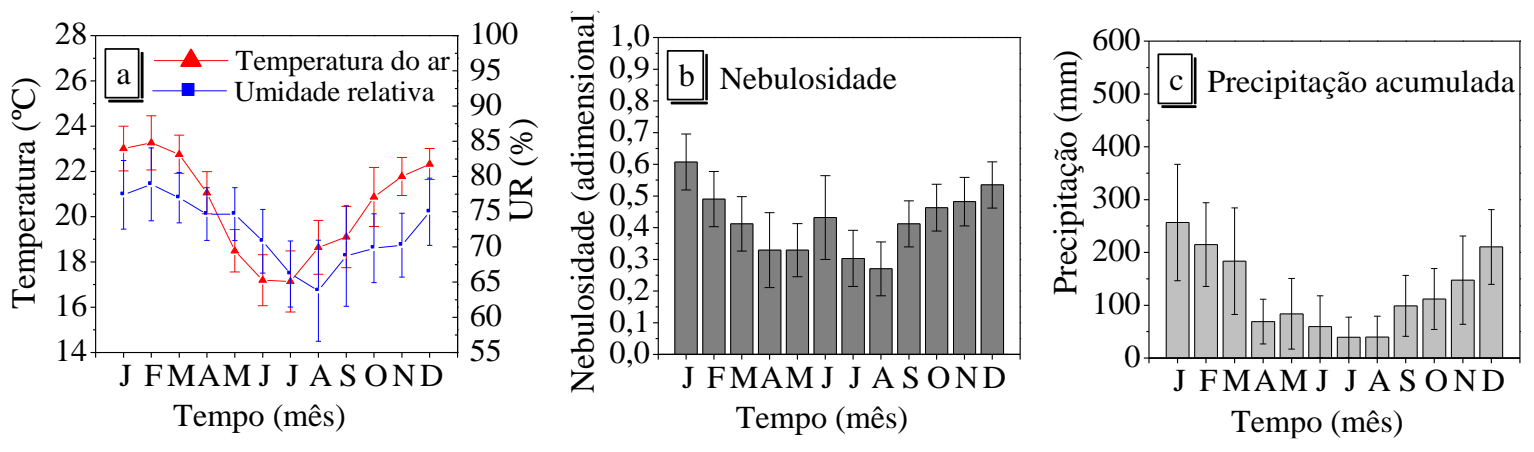

Fonte: Dal Pai et al., (2014).

A evolução anual da precipitação (Fig. 1c) segue a evolução da nebulosidade. É constituída de dois períodos distintos: chuvoso e seco, onde o limite entre os períodos é o valor da precipitação de $100 \mathrm{~mm}$, aproximadamente. No período chuvoso, de outubro a março (primavera/verão) a precipitação é de natureza convectiva, originada do processo de convecção livre. No período seco, de abril a setembro (outono/inverno), a precipitação é do tipo frontal, originada do encontro das massas frias e secas vindas da região sul com as massas quentes e úmidas presentes na região sudeste. A maior precipitação ocorre no mês de janeiro com total de $260,7 \mathrm{~mm}$ e a mínima em agosto com 38,2mm (Dal Pai et al., 2014).

A obtenção dos dados foi realizada em duas frentes: parte dos dados foi obtida por sensoriamento remoto: dados de satélites. Outra parte dos dados foi obtida junto à Estação Meteorológica da UNESP de Botucatu, SP. A base total de dados trabalhados constará com o período de 6 anos: 2010, 2011, 2012, 2013, 2014 e 2015. Embora as bases de dados tenham um período de tempo disponível maior, esse período foi utilizado por ter boa qualidade em todas as suas partições (posteriormente 
será mencionado: são duas bases de dados distintas: uma vinda de satélites e outra base de dados da estação meteorológica local).

Os dados obtidos por sensoriamento remoto foram obtidos na plataforma "Google Engine ${ }^{\circledR}$. Esta plataforma permite obter dados (e imagens) de satélites de maneira rápida, com o acesso da localização via plataforma "Google ${ }^{\circledR}$ " (Gorelick et al, 2017). Todos os dados obtidos neste trabalho foram referentes às coordenadas geográficas da Estação Meteorológica da UNESP de Botucatu, SP (latitude $22^{\circ} 50^{\prime} \mathrm{S}$; longitude $48^{\circ} 25^{\prime}$ W; altitude 786m)(a plataforma "Google Engine" exige uma coordenada de entrada para funcionar). Esta estação meteorológica situa-se dentro do Campus da Unesp-Lageado-Botucatu, onde predomina-se na classificação do uso de solo como "Área Rural", pouco impermeabilizada e com grandes áreas vegetadas (grama), próximo a áreas de produção vegetal (experimentos) e produção animal (junto à FMVZ-Botucatu-SP).

Para o referido trabalho foram obtidas duas bases de dados distintas, junto à plataforma supracitada, com o rigor exigido na metodologia científica (Estrela, C., 2018). Foram escolhidas por fornecer/disponibilizar fluxos de carbono em períodos concomitantes com dados disponíveis em Botucatu. A primeira base utilizada foi a base "PML_V2: Coupled Evapotranspiration and Gross Primary Product" (Zhang et al., 2019; Gan et al., 2018; Zhang et al., 2016). Essa base de dados é disponibilizada nos períodos de julho de 2003 até dezembro de 2017 (lembrando que neste trabalho serão considerados apenas os anos de 2010 a 2015). A segunda base de dados utilizada foi a "MOD17A2H.006: Terra Gross Primary

Productivity 8-Day Global 500M 500 m". (Running \& Zhao, 2019). No seguimento deste trabalho estas bases serão mencionadas como base "PML_V2" e base "MOD17A2H".

As duas bases de dados foram processadas/elaboradas com dados de satélites da NASA (Zhang et al., 2019; Gan et al., 2018; Zhang et al., 2016; Running \& Zhao, 2019). O programa de sensores MODIS - Moderate Resolution Imaging Spectroradiometer - presentes nos satélites "Terra" e "Aqua" da NASA. São base de dados para a elaboração das duas bases PML_V2 e MOD17A2H. Este programa de sensores capta informações do planeta Terra desde o ano de 1999 e oferece cobertura global com a resolução de 8 ou 16 dias dependendo do produto. A resolução gráfica do sensor é de 500m. Cada pixel corresponde a uma área de 500 x 500 metros. Os dados (utilizados neste trabalho) nesta plataforma são disponibilizados a cada 8 dias. A base PML_V2 já vem corrigida na escala diária (porém oferecendo 1 valor a cada 8 dias representando a média do dia e dos sete dias seguintes). A base MOD17A2H vem na escala de 8 dias: para se encontrar o valor diário desta base deve-se dividir seu valor por 8 (no último intervalo do ano de 27 a 31 de dezembro deve-se dividir o valor por 5; divide-se este último valor por 6 em anos bissextos). LEUNING, R., ZHANG, Y.Q., RAJAUD, A., CLEUGH, H., TU, K

A base PML_V2 recebe este nome devido à sua origem no modelo Penman-Monteith-Leuning (Leuning, Zhang, Rajaud, Cleugh \& Tu, 2008). Neste trabalho, Leuning propõe um modelo de se estimar a evapotranspiração "PenmanMonteith" com dados do programa MODIS. Essa estimativa é feita com base em estimativas da condutância estomática, esta estimada com base em medidas de NDVI. Nesta estimativa são oferecidas ferramentas que permitem a estimativa da GPP (Gross Primary Production - produção primária bruta - fotossíntese bruta, em Kg de carbono por $\mathrm{m}^{2}$ dia $^{-1}$ )( Leuning et al., 2008).

A base de dados MOD17A2H recebe este nome por causa do programa de sensores MODIS e sua estimativa é feita com base em modelos da eficiência do uso da radiação solar pelas plantas. É um modelo mais complexo, mas permite a estimativa da respiração e consequentemente da fotossíntese líquida (Nee), que é a fotossíntese bruta (GPP) menos a respiração (R). Esta estimativa é possível pois além de dados de radiação solar global e radiação solar global PAR, o modelo utiliza valores de temperatura do ar, vapor d'água na atmosfera e índices de vegetação (medidos por satélites e mais a utilização de parâmetros locais de vegetação: cada grande bioma mundial apresenta peculiaridades, corrigidas no modelo MOD17A2H)( Zhang et al., 2019; Gan et al., 2018; Zhang et al., 2016). 
Para elaboração dos modelos foram utilizados dados dos anos de 2010 a 2014. A ano de 2015 não foi utilizado na elaboração do modelo. O ano de 2015 foi utilizado para validação dos modelos. Na validação serão utilizados os indicadores estatísticos de acurácia MBE e RMSE:

$$
\begin{aligned}
& M B E=\frac{1}{N} \sum_{i=1}^{N}\left(X_{\text {ESTIMADO }}-X_{\text {OASERVADO }}\right) \\
& M B E(\%)=\left(\frac{M B E}{\overline{X_{\text {OBSERBADO }}}}\right) * 100 \\
& R M S E=\sqrt{\frac{1}{N} \sum_{i=1}^{N}\left(X_{\text {ESTIMADO }}-X_{\text {OBSERVADO }}\right)^{2}} \\
& \operatorname{RMSE}(\%)=\left(\frac{\text { RMSE }}{\overline{X_{\text {OBSERVADO }}}}\right)
\end{aligned}
$$

Dados meteorológicos locais foram obtidos junto à Estação Meteorológica da UNESP de Botucatu, SP. É uma estação meteorológica/climatológica operando desde 1971. É situada dentro de uma escola de agronomia, ambiente rural, com cobertura gramada seguindo o referencial de normas estabelecidos pela W.W.O (World Meteorological Organization, 2008). Para o período do experimento, a base automática foi utilizada. Os dados de radiação solar global foram medidos por um piranômetro Kipp and Zonnen CM3. A radiação solar difusa foi medida com um piranômetro idêntico ao da radiação solar global, porém instalado sob a sobra de um anel de sombreamento, conforme descrito por Melo-Escobedo (Melo \& Escobedo, 1994). A medição da radiação solar difusa pelo método do anel de sombreamento exige correções. A correção isotrópica (geométrica) é feita segundo Melo-Escobedo (Melo \& Escobedo, 1994), enquanto que uma correção anisotrópica feita com base na cobertura do céu por nuvens é feita com base no trabalho de Dal Pai et al., 2016. Na aquisição dos dados foi utilizado um datalogger da marca Campbell CR1000, operando na frequência de 0,2 Hz e armazenando médias de 5 minutos ou 300 segundos. Os valores de radiação solar serão apresentados neste trabalho constarão de médias diárias de 8 dias (exceto último período do ano com 5 dias (6 em anos bissextos)), idênticos aos dados disponíveis nas bases PML_V2 e MOD17A2H.

\section{Resultados e Discussão}

\subsection{Produção diária bruta de carbono - GPP - PML_V2}

Na Figura 2 são apresentados valores de produção diária bruta de carbono (fotossíntese) (GPP), em Kg de carbono assimilados por dia por metro quadrado, da série PML_V2 (Zhang et al., 2019; Gan et al., 2018; Gorelick et al., 2017; Zhang et al., 2016), dados de janeiro de 2010 a dezembro de 2015 : 
Figura 2: Valores de produção diária bruta de carbono (fotossíntese) (GPP), dados de janeiro de 2010 a dezembro de 2015.

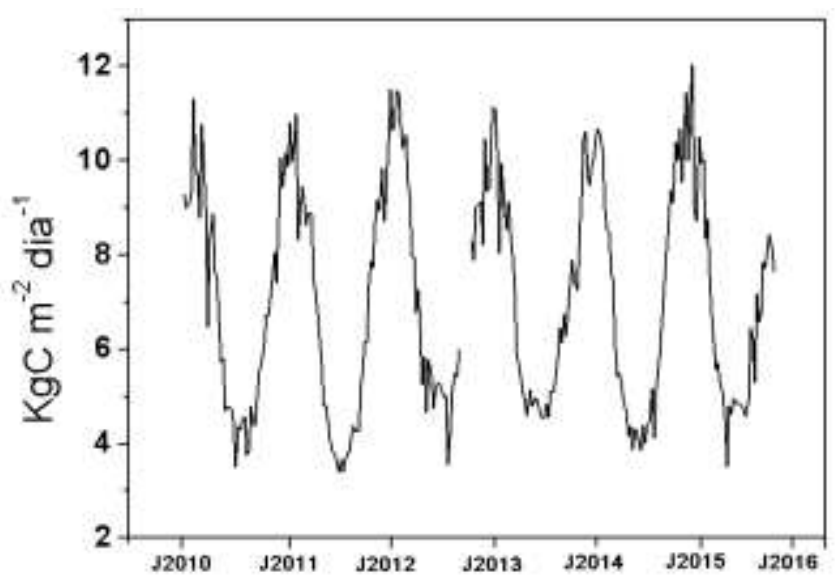

Fonte: Autores (2021).

Os valores da Figura 2 são dados referentes às coordenadas geográficas de Botucatu. Pode-se observar uma sazonalidade nos dados. Maiores valores de produção de carbono em épocas de verão e menores assimilações de carbono em períodos de inverno. Esse comportamento já era esperado pela própria sazonalidade na disponibilidade energética. Há um período sem dados entre o final de 2012 e início de 2013. Essa ausência de dados não é severa, pois se observando a Figura 2 o período de falhas na medida está entre um pico e um vale, representando um comportamento mediano entre dois pontos extremos.

O valor médio da base de dados é de 7,05 $\pm 2,35 \mathrm{KgCm}^{-2} \mathrm{dia}^{-1}$. O valor mínimo assimilado em um dia no período foi de 3,40 $35 \mathrm{KgCm}^{-2} \mathrm{dia}^{-1}$ entre julho e agosto de 2011, enquanto o período de maior assimilação bruta foi em fevereiro de 2015 , com $12,04 \mathrm{KgCm}^{-2} \mathrm{dia}^{-1}$.

\subsection{Produção diária bruta de carbono - MOD17A2H}

Na Figura 3 são apresentados valores de produção diária bruta de carbono (fotossíntese) (GPP), em Kg de carbono assimilados por dia por metro quadrado, da série MOD17A2H (Running \& Zhao, 2019; Gorelick et al., 2017), dados de janeiro de 2010 a dezembro de 2015:

Figura 3: Valores de produção diária bruta de carbono (fotossíntese) (GPP), fotossíntese líquida (Nee) e Respiração (GPPNee), dados de janeiro de 2010 a dezembro de 2015.

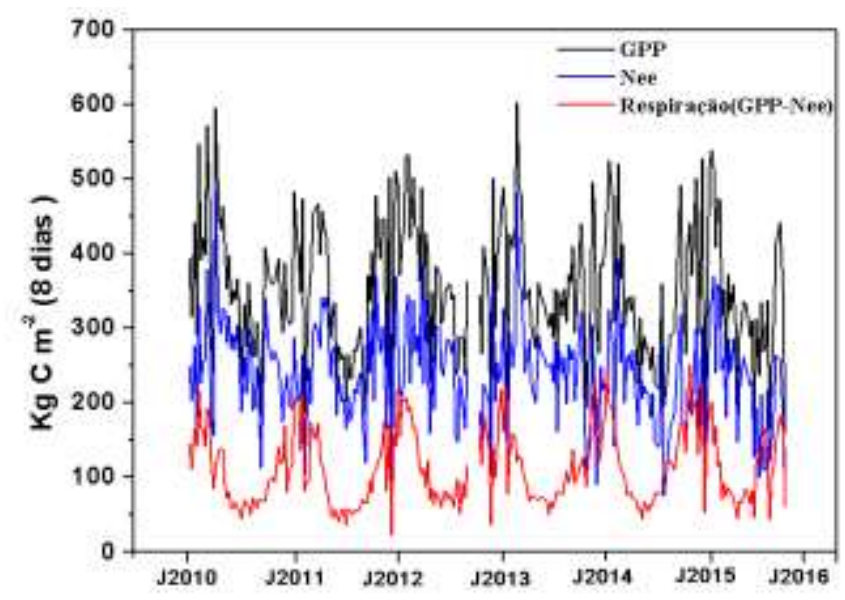

Fonte: Autores (2018). 
Os valores da Figura 3 guardam semelhança em comportamento sazonal com a Figura 2. A Figura 3 (MOD17A2H) apresenta valores indisponíveis na série PML_V2 (Running \& Zhao, 2019): a fotossíntese líquida, e consequentemente a respiração (obtém-se a respiração por diferença entre a produção bruta de carbono e a produção líquida de carbono). Estes valores também apresentam sazonalidade, sendo todos maiores nas épocas de maiores disponibilidades energéticas. A média da GPP nesta série foi de $351,5835 \pm 92.25 \mathrm{KgCm}^{-2} \mathrm{dia}^{-1}$. Observando a GPP da série PML_V2 observa-se grande diferença dos números. Mesmo a base MOD17A2H tendo a escala em oito dias, dividindo-se a média por 8 obtém-se 43,95 $\mathrm{KgCm}^{-2} \mathrm{dia}^{-1}$. É um valor muito superior aos $7 \mathrm{KgCm}^{-2} \mathrm{dia}^{-1}$ da série PML_V2. Essa diferença é devido à metodologia utilizada. A metodologia utilizada na base de dados MOD17A2H utiliza parâmetros de vegetação, o que torna o modelo mais sensível a variações. Na modelagem original, realizada pelo programa "MODIS" da NASA, a região do experimento (Botucatu-SP) é classificada como "Cropland" (plantação).

Existem parâmetros que podem explicar essa variação. O parâmetro LUE - Ligh Use Eficiency - eficiência do uso da luz, dado em Kg de carbono por metro quadrado por dia por $\mathrm{MJ}\left(\mathrm{KgCm}^{-2} \mathrm{dia}^{-1} \mathrm{MJ}^{-1}\right)$, variando de 0,000841 (“Open Shrubland" - em tradução livre (e talvez não recomendável) "Matagal aberto") até 0,001262 para florestas tropicais úmidas (no texto original de Running \& Zhao, 2019, é utilizado o termo “Evergreen Broadleaf Forest - EBF"). Para "Cropland" o seu valor é $0,001044 \mathrm{KgCm}^{-2} \mathrm{dia}^{-1} \mathrm{MJ}^{-1}$. O parâmetro SLA (Projected leaf área per unit mass of leaf carbon - área foliar por unidade de massa de carbono foliar, dado em $\mathrm{m}^{2} \mathrm{Kg} \mathrm{C}^{-1}$, varia de 9 até 37,5 $\mathrm{m}^{2} \mathrm{Kg} \mathrm{C}^{-1}$. Existem mais três parâmetros sobre proporções entre plantas (raiz/parte aérea), que geram mais três parâmetros sobre a respiração da parte aérea, da raiz e da planta inteira. Sugere-se maior estudo na aplicação destes modelos.

O valor médio da GPP foi de $351,58 \pm 92,25 \mathrm{KgCm}^{-2} \mathrm{em} 8$ dias. O valor mínimo assimilado em um dia no período foi de $8325 \mathrm{KgCm}^{-2}$ em 8 dias, enquanto o período de maior assimilação bruta foi $602,25 \mathrm{KgCm}^{-2} \mathrm{em} 8$ dias. Na Tabela 1 são apresentados valores médios da série:

Tabela 1: Média da série MOD17A2H, valores em $\mathrm{KgCm}^{-2} \mathrm{em} 8$ dias:

\begin{tabular}{|c|c|c|c|c|}
\hline & Média & $\operatorname{Max}$ & Min & Soma \\
\hline GPP & $351,58 \pm 92,25$ & 602 & 83 & 90005 \\
\hline Nee & $239,83 \pm 68,86$ & 485 & 60 & 61396 \\
\hline Respiração & $111,75 \pm 49,92$ & 251 & 23 & 28609 \\
\hline
\end{tabular}

Fonte: Autores (2018).

\subsection{Radiação solar Global e Difusa}

Na Figura 4 é apresentada a série obtida junto à Estação Meteorológica da UNESP de Botucatu, SP (latitude 22 $50^{\prime}$ $\mathrm{S}$; longitude $48^{\circ} 25 \mathrm{~W}$; altitude $786 \mathrm{~m}$ ). 
Figura 4: Radiação solar em Botucatu no período de 2010 a 2015. Valores em $\mathrm{MJm}^{-2} \mathrm{dia}^{-1}$.

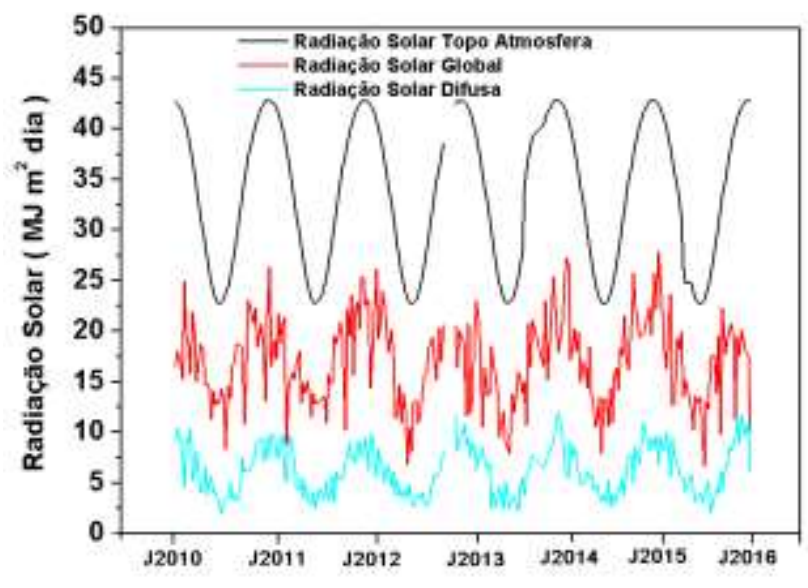

Fonte: Autores (2021).

Os valores da Radiação solar no Topo da atmosfera são calculados e não medidos, conforme Iqbal (1983). Esses valores de Radiação no Topo da atmosfera não apresentam diferenças entre anos diferentes (por ser calculada apenas com a data e a latitude local). As imperfeições em sua série representam períodos em que problemas nas medidas, no equipamento ou no processamento não permitiram a correta aquisição de seus valores (para que os dados fiquem pareados elimina-se valores calculados em dias em que não há medidas). No total foram processados 6 anos de dados, de 2010 a 2015(2010 a 2014 para o modelo e 2015 para a validação). Todos os valores foram processados na mesma escala temporal das bases de dados obtidas de satélites (8 dias). Desconsiderando-se valores espúrios, obteve-se para o modelo (2010-2014) um total de 210 valores (n).

A radiação solar segue a variação astronômica das posições da Terra e do Sol. A radiação solar no topo da atmosfera tende a ser maior no hemisfério sul quando a declinação solar for negativa. O contrário acontece quando a declinação solar se torna positiva: seus valores diminuem. As radiações global e difusa acompanham a variação da radiação no topo da atmosfera. Porém, estas duas radiações: global e difusa, interagem com a atmosfera local. Quanto maior a presença de nuvens (e consequentemente vapor d'água) menor se torna a global. Com o aumento da nebulosidade há uma diminuição da radiação solar direta, aumentando a participação da radiação solar difusa dentro da radiação solar global (radiação solar global = radiação solar direta na horizontal + radiação solar difusa).

Quando se divide a radiação solar global pela radiação solar do topo da atmosfera obtém-se uma relação (fração) que fornece a ideia de transmissividade atmosférica (o que chega na superfície dividido pelo que chega no topo da atmosfera, sendo este valor no topo considerado o valor máximo possível de radiação solar - Kt). Isto porque seu valor expressa bem a nebulosidade: valores abaixo de 0,35 representam sempre um céu nublado sendo a radiação solar global toda difusa. Valores acima de 0,65 representam sempre céu aberto com grande predominância da radiação solar direta na radiação solar global. Valores no meio deste intervalo representam céu parcialmente nublado. Escobedo et al., 2009, propõe duas classificações para o céu nublado: de 0,35 a 0,55 é um céu parcialmente nublado tendendo para o céu nublado (difuso); e de 0,55 a 0,65 um céu parcialmente nublado tendendo para o aberto (direta). Seus valores médios mensais são bons indicadores de nebulosidade. Neste texto a divisão da radiação solar global pela radiação solar no topo da atmosfera será denominada "Kt".

Na Figura 5 é apresentada a média mensal dos valores de Kt durante os 6 anos considerados no experimento. 
Figura 5: Média mensal e desvio padrão dos valores Kt (Hg/Ho) medidos em Botucatu no período de 2010 a 2015.

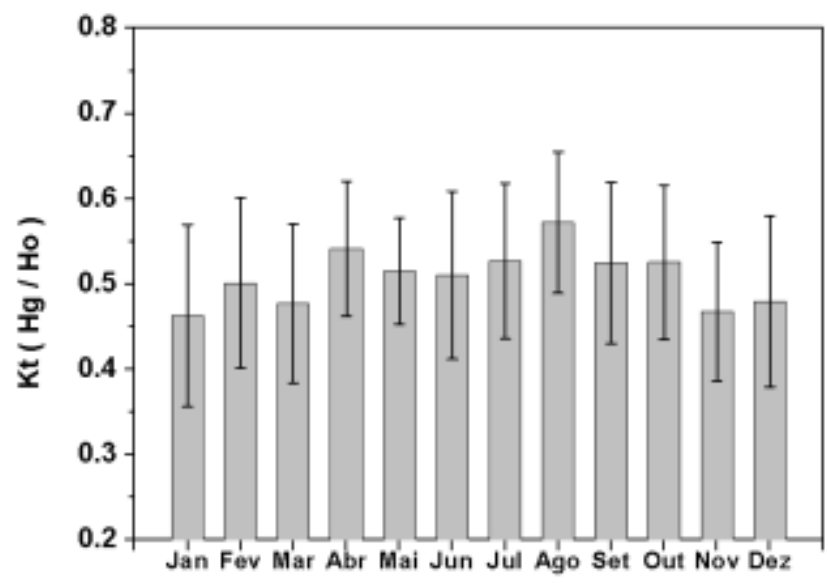

Fonte: Autores (2021).

Baixos valores de Kt indicam para uma cobertura de céu com maior nebulosidade (e presença de água na atmosfera). $\mathrm{O}$ menor valor de kt ocorreu no mês de janeiro, com Kt $=0,463 \pm 0,107$, representando o mês com maior presença de nuvens em Botucatu. O mês de agosto apresentou Kt de 0,572 $\pm 0,083$, representando o mês com menor nebulosidade. Isto é importante pois a radiação solar difusa é muito modificada pela presença/ausência de nebulosidade. O valor Kt médio em Botucatu foi de 0,509 \pm 0,033(valores obtidos com dados de 2010 a 2015, valor este muito próximo do trabalho de Escobedo et al., 2009, que encontrou para a mesma localidade um valor de $\sim 0,50$ ).

Outra útil relação é a da radiação solar difusa pela radiação solar global. Esta divisão (chamada de "Kd") representa a fração da participação da radiação solar difusa dentro da radiação solar global. Quando esta relação Kd fica mais elevada representa uma elevação na quantidade de radiação solar difusa, indicando menor presença de nuvens. A Figura 6 apresenta valores de Kd mensais calculados durante o experimento em Botucatu-SP:

Figura 6: Média mensal e desvio padrão dos valores $\mathrm{Kd}(\mathrm{Hd} / \mathrm{Hg})$ medidos em Botucatu no período de 2010 a 2015.

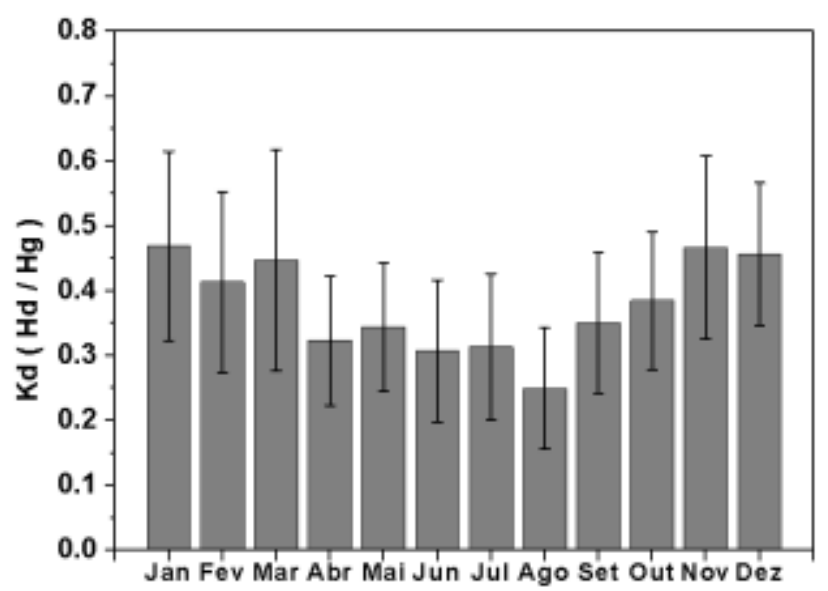

Fonte: Autores (2021).

Observa-se o maior valor no mês mais nebuloso: Janeiro, com Kd representando 0,469 $\pm 0,146$. O mês com menos participação da difusa na global é agosto, com Kd igual a 0,249 $\pm 0,093$. A média total é de 0,377 $\pm 0,073$. 


\subsection{Relação Radiação Solar Difusa com a GPP(PML_V2)}

Na Figura 7 pode-se observar a evolução da radiação solar difusa e a evolução sazonal do fluxo de carbono bruto (GPP - PML_V2) produzido pelas plantas:

Figura 7: Evolução dos fluxos de GPP e da radiação solar difusa (valores diários) no período de 2010 a 2015.

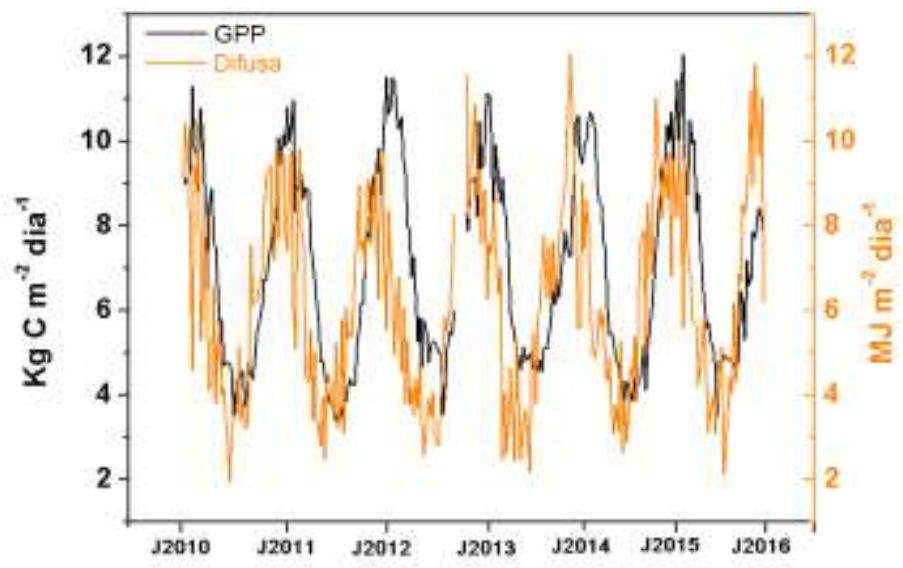

Fonte: Autores (2021).

Nota-se uma semelhança entre as curvas da GPP e da radiação solar difusa. Na Figura 7 poderia ter-se utilizado a mesma escala y (em valores numéricos, as unidades não são as mesmas). A semelhança era esperada pois o modelo da GPP é baseado em modelos de fluxo de energia no ambiente (Penman). O que chama a atenção é o fato de que cada linha da Figura veio de uma fonte diferente. A linha laranja da radiação solar difusa foi obtida com medidas na superfície do planeta. A linha escura da GPP veio do sensoriamento remoto. Pode-se dizer que algo parecido foi observado pelas duas técnicas.

Na Tabela 2 são apresentados valores médios separados por meses, no período de 2010 a 2015:

Tabela 2: Valores médios mensais do período de 2010 a 20015.

\begin{tabular}{|c|c|c|c|c|}
\hline \multicolumn{2}{|r|}{ Ho $\left(\mathrm{MJ} / \mathrm{m}^{2}\right)$} & $\mathrm{Hg}\left(\mathrm{MJ} / \mathrm{m}^{2}\right)$ & $\operatorname{Hd}\left(\mathbf{M J} / \mathbf{m}^{2}\right)$ & \multirow{2}{*}{$\begin{array}{c}\mathrm{GPP}\left(\mathrm{KgC} / \mathrm{m}^{2} \text { dia }\right) \\
\text { Média }\end{array}$} \\
\hline & & Média & Média & \\
\hline $\mathbf{J}$ & 42.25 & $19.59 \pm 4.48$ & $8.60 \pm 1.48$ & $9.680 \pm 0.696$ \\
\hline $\mathbf{F}$ & 39.66 & $19.89 \pm 3.98$ & $7.76 \pm 1.71$ & $10.396 \pm 0.934$ \\
\hline $\mathbf{M}$ & 34.77 & $16.62 \pm 3.45$ & $6.93 \pm 1.49$ & $9.697 \pm 1.096$ \\
\hline $\mathbf{A}$ & 29.31 & $15.87 \pm 2.52$ & $4.96 \pm 1.15$ & $8.635 \pm 0.621$ \\
\hline M & 25.37 & $13.09 \pm 1.79$ & $4.37 \pm 0.93$ & $6.629 \pm 0.777$ \\
\hline $\mathbf{J}$ & 22.80 & $11.63 \pm 2.26$ & $3.40 \pm 0.79$ & $4.991 \pm 0.454$ \\
\hline $\mathbf{J}$ & 24.17 & $12.76 \pm 2.36$ & $3.78 \pm 0.89$ & $4.411 \pm 0.624$ \\
\hline A & 28.96 & $16.45 \pm 2.16$ & $3.95 \pm 0.95$ & $4.422 \pm 0.547$ \\
\hline $\mathbf{S}$ & 34.71 & $18.18 \pm 3.15$ & $6.09 \pm 1.09$ & $4.648 \pm 0.609$ \\
\hline $\mathbf{O}$ & 38.36 & $20.17 \pm 3.45$ & $7.45 \pm 1.10$ & $5.595 \pm 0.760$ \\
\hline $\mathbf{N}$ & 41.19 & $19.27 \pm 3.36$ & $8.66 \pm 1.67$ & $6.876 \pm 0.650$ \\
\hline D & 42.71 & $20.49 \pm 4.28$ & $8.99 \pm 1.48$ & $8.486 \pm 0.871$ \\
\hline & $33.69 \pm 7.33$ & $16.99 \pm 3.13$ & $6.25 \pm 2.08$ & $7.04 \pm 2.54$ \\
\hline
\end{tabular}

Fonte: Autores (2021). 


\subsection{Relação entre a Radiação Solar Difusa e a GPP:}

$\mathrm{Na}$ tentativa de se encontrar uma equação para se estimar fluxos de $\mathrm{CO}_{2}$, a relação entre a radiação solar difusa e a GPP deve ter a radiação solar difusa como variável independente (x) e GPP como a variável dependente. O modelo linear é apresentado na Figura 8. Para o modelo foram utilizados dados dos anos de 2010 a 2014 . O ano de 2015 foi utilizado para validação.

Figura 8: Relação entre a radiação solar difusa e a GPP. A linha vermelha é o melhor ajuste linear $y=a+b * x: a=3,881$ e $b=$ 0,$5161 ; \mathrm{r}=0,5147 ; \mathrm{n}=210$.

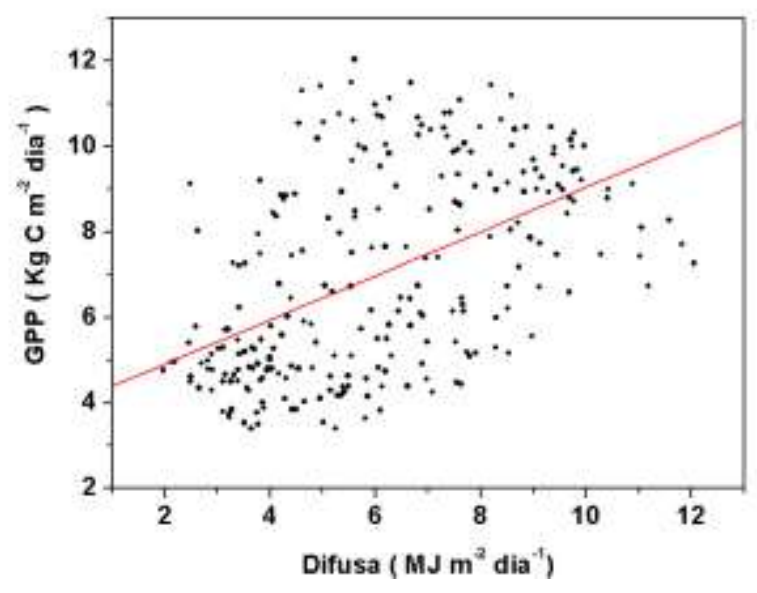

Fonte: Autores (2021).

No modelo linear com a difusa no eixo x e a GPP no eixo Y, temos a linha vermelha sendo o modelo linear. Com o ajuste linear não passando pela origem temos o termo angular valendo $0,5161 \mathrm{e}$ o termo linear valendo 3,881 . Nesta tentativa o $\mathrm{r}$ indica baixo desempenho do modelo. Para se obter este "melhor ajuste linear", o termo linear não foi nulo, representando outra imperfeição no modelo: quando a difusa tiver valor zero (ou muito baixo), o valor de produção primária tenderá ao termo linear: $3,881 \mathrm{KgCm}^{-2} \mathrm{dia}^{-1}$.

Para o modelo multivariado as variáveis de entrada utilizadas foram a radiação solar difusa, o valor Kt e o mês. O Kt e o mês entraram também no modelo na tentativa de se ponderar a cobertura do céu e a época do ano que a estimativa é feita. Na modelagem foram utilizados os anos de 2010 a 2014: cinco anos no total. O ano de 2015 foi guardado para a validação do modelo. A equação multivariada ajustada, com base na metodologia da redução da soma dos resíduos está representada na Equação:

$G P P=(P 1)+(P 2 * D I F)+\left(P 3 * D I F^{2}\right)+(P 4 * K T)+\left(P 5 * K T^{2}\right)+(P 6 * M E S)+\left(P 7 * M E S^{2}\right)$

GPP: fotossíntese bruta, em $\mathrm{Kg}$ de C por metro quadrado por dia;

DIF: radiação solar difusa, em $\mathrm{MJ} / \mathrm{m}^{2}$ dia;

Kt: relação $\mathrm{Hg} / \mathrm{Ho}$, adimensional (fração);

Mês: mês, de 1 a 12 .

P1 a P7: parâmetros da equação, disponíveis na Tabela 3: 
Tabela 3: Parâmetros da equação multivariada.

\begin{tabular}{|c|c|c|c|c|c|c|}
\hline P1 & P2 & P3 & P4 & P5 & P6 & P7 \\
\hline 4,71485 & 0,52485 & $-0,04245$ & 29,57052 & $-26,91533$ & $-2,35036$ & 0,15255 \\
\hline
\end{tabular}

Fonte: Autores (2021).

Os parâmetros da equação foram obtidos com $\mathrm{r}^{2}$ igual a 0,76742, e um valor chi-quadrado de 1,3296.

$\mathrm{Na}$ validação utilizou-se os valores de radiação solar difusa, Kt e os meses do ano de 2015 para se estimar com a equação acima os valores de GPPest. O resultado da comparação desta estimativa com os valores originais PML_V2 está na Figura 9 (o n para a validação foi $n=46$ ):

Figura 9: Comparação da GPP PML_V2 com a GPP estimada. Valores de GPP em Kg de carbono assimilado por metro quadrado por dia. No Eixo x os meses do ano de 2015, ano reservado para a validação.

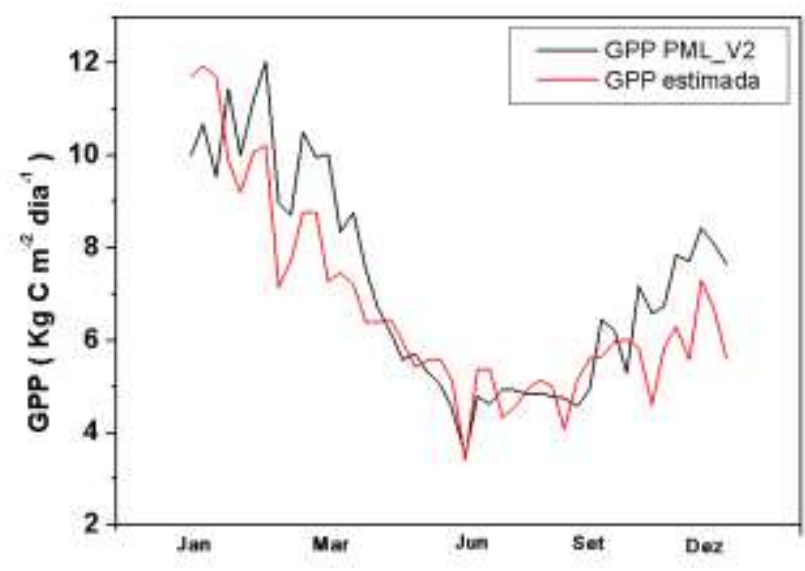

Fonte: Autores (2021).

Observa-se um comportamento similar ao se observar a GPP original PML_V2 e a GPP estimada. Esta estimativa oferecerá a oportunidade de se estimar o fluxo de carbono com base no conhecimento da radiação solar difusa e global. O modelo é válido para o local do experimento, mas pode servir para outras localidades, com esperado menor desempenho (o quanto menor dependerá de mais estudos). Mesmo assim deve-se lembrar que o fluxo de carbono é um elemento ainda pouco medido, devido ao elevado custo do equipamento, sem contar com sua própria elevada complexidade. Bases de dados de radiação solar global e difusa não são tão abundantes quanto séries de temperatura ou chuva, mas começam a se tornar mais acessíveis. O INMET - Instituto Nacional de Meteorologia (www.inmet.org.br) disponibiliza gratuitamente valores da radiação solar global. A radiação solar difusa pode ser estimada com modelos de Kt, como proposto por Escobedo, Gomes, Oliveiras \& Soares (2009).

A comparação direta da GPP da série PML_V2 e a GPP estimada é apresentada na Figura 10: 
Figura 10: Comparação da GPP PML_V2 com a GPP estimada. Valores de GPP em $\mathrm{KgCm}^{-2} \mathrm{dia}^{-1}$. No Eixo x o ano de 2015, ano reservado para a validação.

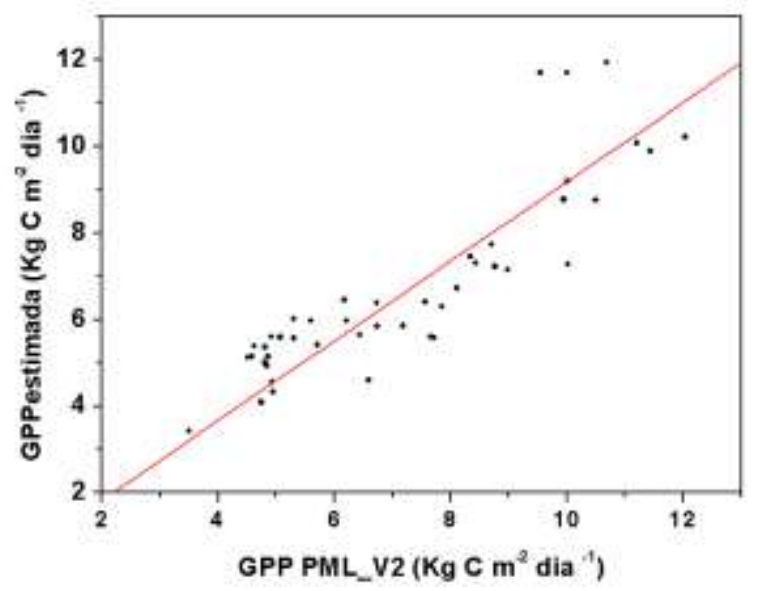

Fonte: Autores (2021).

Na comparação direta entre as duas séries de GPP observa-se bom ajuste. A equação de ajuste da Figura 10 (reta vermelha) é $y=0.91839^{*} x$, com um $\mathrm{r}$ igual a 0.87 . Este resultado sugere que o valor da GPP estimado é em média, aproximadamente, $8 \%$ menor em relação à GPP PML_V2.

$\mathrm{O}$ valor do MBE foi de $-0.504 \mathrm{KgCm}^{-2} \mathrm{dia}^{-1}$, em porcentagem $-6,99 \%$. Pelo modelo de MBE, que tende a indicar o erro simples, o valor -0.504 significa que em média os valores estimados são $0,504 \mathrm{KgCm}^{-2} \mathrm{dia}^{-1}$ menores em relação aos dados observados (PML_V2), significando um valor em média 6,99\% menor. Pelo modelo do erro simples o erro ficou próximo ao confrontamento direto de da GPP estimada com a PML_V2: GPP estimada 8\% menor em relação à GPP PML_V2.

$\mathrm{O}$ valor RMSE foi $1,202 \mathrm{KgCm}^{-2} \mathrm{dia}^{-1}$. O valor RMSE é dotado do comportamento quadrático sob diferenças de valores. Valores muito próximos (abaixo de zero) se aproximam quando elevados ao quadrado, enquanto valores acima de zero se distanciam quando elevados ao quadrado, dando a noção do espalhamento dos pontos ao redor da média. Em porcentagem $16,70 \%$, lembrando que no RMSE o sinal negativo sempre é perdido, representando o valor apenas o espalhamento dos pontos.

\section{Conclusão}

É possível se estabelecer relações entre dados do programa MODIS com dados medidos na superfície, lembrando que nestas comparações existe uma grande diferença instrumental e metodológica.

- A produção diária bruta de carbono, a GPP apresenta relação com a radiação solar difusa, porém ainda carecendo maior número de estudos para se obter melhor compreensão dessa relação.

- Os dados de GPP obtidos junto ao programa "PML_V2" se ajustaram melhor aos dados de radiação solar de Botucatu-SP. Porém não se pode afirmar que o programa "MODIS17A2H" tenha apresentado uma má performance, apenas carece de mais estudos, principalmente em sua parametrização, que envolve elementos de vegetação local.

- A equação multivariada obtida para estimativa da GPP em função da radiação solar difusa, o valor Kt o mês apresentou elevados valores de performance, com $r=0.88, \mathrm{MBE}=-6,99 \%$ e RMSE $=16,70 \%$.

Há necessidade de estudos mais aprofundados sobre os valores de produtividade vegetal na relação entre radiação solar difusa e fluxos de $\mathrm{CO}_{2}$ em futuros estudos. 


\section{Referências}

Alton, P. B., P. \& North, S. O. Los. (2007). The impact of diffuse sunlighton canopy light-use efficiency, gross photosynthetic product and netecosystem exchange in three forest biomes, Global Change Biol., 13, 1-12.

Araújo, T. M., Higuchi, N., Carvalho Jr., J. A. (1996). Comparação de métodos para determinar biomassa na região amazônica. Anais da Academia Brasileira de Ciencias, 68, 39-40. Recuperado de http://hdl.handle.net/11449/64973.

Brodersen, C. R., T. C. Vogelmann, W. E. Williams, \& H. L. Gorton. (2008). A new paradigm in leaf-level photosynthesis: Direct and diffuselight are not equal. Plant Cell Environ. 31, 159-164.

Dal Pai, A., Escobedo, J. F. Correa, Dal Pai, E., Santos M. C. (2014). Estimation of Hourly, Daily and Monthly mean Diffuse Radiation Based Shadowring Correction. Energy Procedia, 57, 1150-1159.

Erbs, D.G., Klein, S.A., Duffie, J.A. (1982). Estimation of the diffuse radiation fraction for hourly, daily and monthly-average global radiation. Solar Energy, 28, 293-302.

Escobedo, J. A., Gomes, E. N., Oliveira, A. P. \& Soares, J. (2009). Modeling hourly and daily fractions of UV, PAR and NIR to global solar radiation under various sky conditions at Botucatu, Brazil. Applied Energy. Oxford: Elsevier, 86(3), 299-309. http://hdl.handle.net/11449/41851.

Estrela, C. (2018). Metodologia Científica: Ciência, Ensino, Pesquisa. Editora Artes Médicas.

Freedman, J. M., D. R. Fitzjarrald, K. E. Moore, \& R. K. Sakai. (2001). Boundary layer clouds and vegetation-atmosphere feedbacks, J. Clim., 14 , $180-197$.

Gan, R., Zhang, Y. Q., Shi, H., Yang, Y. T., Eamus, D., Cheng, L., Chiew, F. H. S., Yu, Q. (2018). Use of satellite leaf area index estimating evapotranspiration and gross assimilation for Australian ecosystems. Ecohydrology, 11(5). Recuperado de: https://doi.org/10.1002/eco.1974.

Gorelick, N., Hancher, M., Dixon, M., Ilyushchenko, S., Thau, D., \& Moore, R. (2017). Google Earth Engine: Planetary-scale geospatial analysis for everyone. Remote Sensing of Environment.

Gu, L., J. D. Fuentes, H. H. Shugart, R. M. Staebler, \& T. A. Black.(1999). Responses of net ecosystem exchanges of carbon dioxide tochanges in cloudiness: Results from two North American deciduous forests, J. Geophys. Res., 104(31), 421-434. 1999.

Instituto Nacional de Meteorologia - INMET - https://portal.inmet.gov.br - 2021.

Iqbal, M. An introduction to solar radiation. Academic Press, 1983. 390p.

Jacovides, C. P., Assimakopoulos, V. D., \& Tymvios, F. S. (2006). Solar global UV radiation and its relationship with solar global radiation measured on the island of Cyprus. Energy, 31, 2728-2738.

Jacovides, C. P., Tymvios, F. S., Assimakopoulos, V. D., \& Kaltsounides, N. A. (2007). The dependence of global and diffuse PAR radiation components on sky conditions at Athens, Greece. Agricultural and Forest Meteorology, 143, 277-287.

Jing, X., Huang, J., Wang, G., Higuchi, K., Bi, J., Sun, Y., Yu, H., \& Wang, T. (2010). The effects of clouds and aerosols on net ecosystem CO 2 exchange over semi-arid Loess Plateau of Northwest China, Atmos. Chem. Phys., 10, 8205-8218. https://doi.org/10.5194/acp-10-8205-2010.

Leuning, R., Zhang, Y.Q., Rajaud, A., Cleugh, H., \& Tu, K. (2008). A simple surface 727 conductance model to estimate regional evaporation using MODIS leaf area index and 728 the Penman-Monteith equation. Water Resour. Res, 44. 10.1029/2007WR006562.

Matthew G. Letts, Peter M. Lafleur \& Nigel T. \& Roulet, (2005). On the relationship between cloudiness and net ecosystem carbon dioxide exchange in a peatland ecosystem, Écoscience, 12:1, 53-69, 10.2980/i1195-6860-12-1-53.1.https://doi.org/10.2980/i1195-6860-12-1-53.1 2005.

Melo, J. M. D., \& Escobedo, J. F.(1994). Medida da radiação solar difusa. In: ENERGÍAS LÍMPIAS EN PROGRESO, VII CONGRESSO IBÉRICO DE ENERGIA SOLAR, Vigo, Espanha. Anais INTERNATIONAL SOLAR ENERGY SOCIETY, 1.

Min, Q., S. Wang. (2008). Clouds modulate terrestrial carbon uptake in amid latitude hardwood forest, Geophys. Res. Lett., 35, 10.1029/2007GL032398.

Oliphant, A. J., D. Dragoni, B. Deng, C.S.B. Grimmond, H.-P. \& Schmid, S.L. Scott. (2011). The role of sky conditions on gross primary production in a mixed deciduous forest. Agricultural and ForestMeteorology, 151(7), 781-791, ISSN 0168-1923. https://doi.org/10.1016/j.agrformet.2011.01.005 (http://www.sciencedirect.com/science/article/pii/S0168192311000244).

Pallardy, S. G. (2008). Chapter 9 - Nitrogen Metabolism. Physiology of Woody Plants (Third Edition), 233-254.

Ren, X., He, H., Zhang, L., \& Yu, G. (2018). Global radiation, photosynthetically active radiation, and the diffuse component dataset of China, 1981-2010, Earth Syst. Sci., 10, 1217-1226. https://doi.org/10.5194/essd-10-1217-2018.

Roderick, M. L., G. D. Farquhar, S. L. Berry, \& I. R. Noble, (2001). On the direct effect of clouds and atmospheric particles on the productivity and structure of vegetation, Oecologia, 129, 21-30.

Running, Steven W., \& Zhao, M, (2019). User's guide daily GPP and annual npp (MOD17A2H/A3H) and year-end gap filled (MOD17A2HGF/A3HGF) products NASA earth observing system MODIS land algorithm (for collection 6). Version 4.2. 10/Junho/2019.

Salisbury, F. B., \& Ross, C. W. (2012). Fisiologia de Plantas - Tradução da $4^{\mathrm{a}}$ edição norte-americana. Cengagelearning, 774p. 
Research, Society and Development, v. 10, n. 13, e342101321307, 2021

(CC BY 4.0) | ISSN 2525-3409 | DOI: http://dx.doi.org/10.33448/rsd-v10i13.21307

Yanni G., Guirui Y., Huimin Y., Xianjin Z., Shenggong L., Qiufeng W., Junhui Z., Yanfen W., Yingnian L., Liang Z., \& Peili S. (2014). A MODIS-based Photosynthetic Capacity Model to estimate gross primary production in Northern China and the Tibetan Plateau. Remote Sensing of Environment. 148, 108118. ISSN 0034-4257. https://doi.org/10.1016/j.rse.2014.03.006. 2014.

Yamashita, M., \& Yoshimura M. (2019). Estimation of Global and Diffuse Photosynthetic Photon Flux Density under Various Sky Conditions Using GroundBased Whole-Sky Images. Remote Sens., 11(8), 932. https://doi.org/10.3390/rs11080932.

Taiz, L., \& Zeiger, E. (2016). Fisiologia vegetal. 6. ed., Artmed, 918 p.

World Weather Organization - WWO - www.wwo.org. 2021.

Zhang X, Zhang Y, \& Zhoub Y. (2000). Masuring and modeling photosynthetically active radiation in Tibet Plateau during April-October. Agricultural Meteorology, 102, 207-212.

Zhang, Y., Kong, D., Gan, R., Chiew, F. H. S., Mcvicar, T. R., Zhang, Q., \& Yang, Y. (2019). Coupled estimation of 500m and 8-day resolution global evapotranspiration and gross primary production in 2002-2017. Remote Sens. Environ, 222, 165-182. https://doi.org/10.1016/j.rse.2018.12.031.

Zhang, Y., Peña-Arancibia, J. L., Mcvicar, T. R., Chiew, F. H. S., Vaze, J., Liu, C., Lu, X., Zheng, H., Wang, Y., Liu, Y. Y., Miralles, D. G., \& Pan, M. (2016). Multi-decadal trends in global terrestrial evapotranspiration and its components. Sci. Rep, 6. https://doi.org/10.1038/srep19124. 